\title{
Influência de variáveis meteorológicas na ocorrência de pneumonia
}

\section{Influence of meteorological variables on the occurrence of pneumonia}

\author{
Enyedja Kerlly Martins de Araújo \\ Carvalho $^{1 \mathrm{i}}$
}

Orcid: http://orcid.org/0000-0002-3031-5922

\author{
Renilson Targino Dantas 2 ii \\ Orcid: http://orcid.org/0000-0002-3982-3704
}

\author{
José Ribamar Marques de Carvalho ${ }^{3 \mathrm{iii}}$ \\ Orcid: http://orcid.org/0000-0003-3482-9231
}

\begin{abstract}
Resumo
As causas da influência do tempo e do clima sobre os seres vivos não são completamente conhecidas, logo é de grande importância os estudos que os relacionam com a saúde humana e as estratégias relacionadas à formulação de políticas públicas de saúde. Esse estudo objetivou analisar a influência das variáveis meteorológicas (precipitação, temperatura e umidade relativa do ar) e o número de casos acometidos por pneumonia no município de Campina Grande, PB no período de 1998 a 2016. Para tanto, foi realizado um estudo epidemiológico, retrospectivo e descritivo com abordagem quantitativa. As principais contribuições configuram-se como fatores essenciais para compreender as consequências que as mudanças nas variáveis climáticas podem ocasionar na saúde da população do município estudado. Como recomendação, destaca-se a limitação do estudo que se relaciona às subnotificações das doenças, diagnósticos incorretos, dentre outros, que podem ocorrer junto às repartições responsáveis pelo fornecimento de informações de saúde.
\end{abstract}

Palavras-chave: Clima. Saúde. Variáveis meteorológicas.

\begin{abstract}
The causes of the influence of time and climate on living beings are not completely known, so studies that relate them to human health and strategies related to the formulation of public health policies are of great importance. This study aimed to analyze the influence of meteorological variables (precipitation, temperature and relative humidity) and the number of cases affected by pneumonia in the municipality of Campina Grande, PB in the period from 1998 to 2016. For this purpose, an epidemiological study was carried out, retrospective and descriptive with quantitative approach. The main contributions are configured as essential factors to understand the consequences that changes in climatic variables can have on the health of the population of the studied municipality. As a recommendation, the limitation of the study that relates to the underreporting of diseases, incorrect diagnoses, among others, which may occur with the departments responsible for providing health information, stands out.
\end{abstract}

Keywords: Climate. Health. Meteorological variables.

\footnotetext{
${ }^{1}$ Faculdade São Francisco da Paraíba. Paraíba - Brasil. E-mail: enyedjkm@gmail.com

${ }^{2}$ Universidade Federal de Campina Grande - Paraíba - Brasil. E-mail: renilson@dca.ufcg.edu.br

${ }^{3}$ Universidade Federal de Campina Grande - Paraíba - Brasil. E-mail: profribamar@gmail.com
} 


\section{INTRODUÇÃO}

As preocupações com a problemática ambiental e suas relações estão inseridas na Saúde Pública desde seus primórdios, apesar de só na segunda metade deste século, ter se estruturado como uma área típica para abordar, debater e discutir essas questões com vistas a identificar interrelações entre saúde e meio ambiente, passando a ser denominada pela Organização Mundial da Saúde (OMS) como Saúde Ambiental.

Assim, em sua definição mais ampla, o campo da saúde ambiental compreende a área da saúde pública afeita ao conhecimento científico e à formulação de políticas públicas relacionadas à interação entre a saúde humana e a criação e implementação de programas de vigilância em saúde ambiental em vários países do mundo, incluindo o Brasil (BRASIL, 2010).

No entanto, entende-se que as mudanças climáticas interferem diretamente no ambiente colocando em risco o equilíbrio ecológico do planeta, influenciando entre outras coisas no surgimento de doenças e na qualidade de vida da população.

Nas últimas décadas o estudo dos efeitos do clima na saúde da população vem sendo divulgado e discutido pela mídia, por instituições, pesquisadores, especialistas de diversas áreas do conhecimento. As alterações nos elementos meteorológicos, como temperatura e umidade relativa do ar e precipitação podem trazer maior abundância e disseminação de vetores e patógenos. Portanto, as anomalias climáticas associadas a tais fenômenos tendem a ser catastróficas e provocar prejuízos sociais, econômicos e ambientais.

As causas da influência do tempo e do clima sobre os seres vivos não são completamente conhecidas, logo é de grande importância os estudos que os relacionam com a saúde humana e as estratégias relacionadas à formulação de políticas públicas de saúde. Notadamente, as doenças respiratórias são muito comuns e as formas de contraí-las também são muito abrangentes, por isso torna-se imprescindível realizar estudos regionalizados, além de identificar possíveis fatores, como as alterações climáticas. Nesse sentido, a discussão desse assunto tem sido ampliada por parte dos profissionais das mais variadas áreas, para que sejam estabelecidas relações entre a alteração das variáveis meteorológicas e o surgimento de doenças.

A importância de pesquisar os condicionantes responsáveis pela manifestação de doenças respiratórias, especialmente de pneumonia, decorre do fato de que essa doença sofre influência direta ou indireta de elementos meteorológicos e socioambientais (clima, urbanização e modo de vida das populações).

A OMS divulgou que a pneumonia é a que mais mata crianças menores de cinco anos, sendo a estimativa de 1,2 milhão em todo o mundo, mais do que os óbitos provocados pela AIDS, malária e tuberculose reunidas. Desses óbitos por pneumonia, mais de $99 \%$ seriam registrados em países em desenvolvimento como o Brasil, o que faz com que a OMS tenha reforçado o pedido a esses governos de dar prioridade à prevenção e ao combate à doença. De acordo com essa organização, a pneumonia é um dos problemas com maior possibilidade de solução no cenário da saúde global (WHO, 2014).

Nesse aspecto, as variáveis climáticas têm gerado preocupação crescente quanto aos potenciais efeitos à saúde humana, especialmente aqueles relacionados às doenças transmissíveis, pois constituem importante causa de morbimortalidade e afligem milhões de pessoas em diferentes regiões do mundo, especialmente em países subdesenvolvidos. Constituindo as variações climáticas, um enorme contribuinte para o agravo de doenças, o conhecimento das 
condições meteorológicas favorece a prevenção de danos para a sociedade, seja de ordem material ou humana. Visto que, as alterações climáticas interferem diretamente no ambiente colocando em risco o equilíbrio ecológico do planeta, influenciando entre outras coisas no surgimento de doenças e na qualidade de vida da população.

Vários têm sido os estudos que procuram investigar essa temática, seja no âmbito nacional (SILVA JÚNIOR, 2011; MURARA, MENDONÇA \& BONETTI, 2013; COSTA et al. 2014; SILVA et al. 2016; SANTOS et al. 2016; PONTES et al. 2016; MANDÚ et al. 2019), como no âmbito internacional (SALVI, 2007; OLULEYE \& AKINBOBOLA, 2010; RUDAN et al. 2013; QIU et al. 2016; GHALHARI \& MAYVANEH, 2016) que evidenciam sua relevância para o entendimento dos condicionantes relacionados às variáveis climáticas e os casos de pneumonia reforçando a importância para fortalecer os estudos regionalizados em contextos geográficos específicos e contribuir para a gestão e formulação de políticas públicas que possam minimizar impactos sociais, econômicos e ambientais da população.

Nessa perspectiva, o presente estudo procura responder ao seguinte questionamento: Qual a influência das variáveis meteorológicas (temperatura e umidade relativa do ar e precipitação) e a incidência de pneumonia na cidade de Campina Grande, PB?

Para tanto, objetiva analisar a influência das variáveis meteorológicas (precipitação, temperatura e umidade relativa do ar) no número de casos acometidos de pneumonia no município de Campina Grande, PB, no período de 1998 a 2016.

\section{REVISÃO DA LITERATURA}

\section{Interações entre Clima, Saúde Ambiental e Saúde Humana}

Segundo a Secretaria de Vigilância em Saúde do MS, na Instrução Normativa 01/05, Saúde Ambiental compreende a área da saúde pública afeita ao conhecimento científico e à formulação de políticas públicas relacionadas com a interação entre a saúde humana e os fatores do meio ambiente natural e antropogênico que a determinam, condicionam, no intuito de melhorar a qualidade de vida do ser humano sob o ponto de vista da sustentabilidade (MOTA, 2013).

Para Mota (2013) a saúde ambiental constitui, portanto, a área da saúde pública que considera os efeitos que o meio ambiente pode exercer sobre o bem-estar físico, mental e social do ser humano, ou seja, que associa as condições do meio à saúde da população.

Conforme Amorim et al. (2013) há tempos, estudos correlacionam a influência do meio ambiente sobre o organismo humano, tentando compreender o complexo processo de saúde e doença, resultante da ação da sociedade em face da apropriação da natureza e organização do espaço.

Dentre todas as preocupações sobre os impactos causados pela degradação ambiental, a mais complicada envolve a perda da qualidade de vida da população. Dessa forma, entende-se que a preocupação em tecer relações entre ambiente e saúde se refere muito mais a tentar realizar denúncias sobre a qualidade de vida e demonstrar que o clima é apenas uma variável, e que hoje as doenças, antes de qualquer outro fator, são determinadas socialmente.

Um grande instrumento de obtenção das mais variadas formas de conhecimentos é a meteorologia e a climatologia, permitindo à humanidade um entendimento prévio do passado e presente sobre o clima e sua dinâmica (CONCEIÇÃO et al., 2015).

O clima, entre outros fatores, pode suscitar a manifestação de determinadas doenças à saúde através de suas propriedades (a temperatura e umidade relativa do ar, precipitação pluviométrica, 
pressão atmosférica e ventos), que interferem no bem-estar das pessoas (SANTOS et al., 2016).

Para tanto, os dados da OMS (2015) apontam que o clima tem papel fundamental na transmissão de diversas doenças que estão entre as principais causas de morbidade e mortalidade no mundo. Sendo um importante agente de disseminação de diversas doenças, as variáveis meteorológicas (temperatura do ar, precipitação pluviométrica e umidade relativa do ar) são importantes objetos de investigação.

De acordo com Rouquayrol e Almeida Filho (2013) no campo epidemiológico, o clima é o aspecto do ambiente físico que tem até agora concentrado maior atenção para estudos epidemiológicos, sendo a resultante de toda variedade de fenômenos meteorológicos específicos, que caracterizam a situação média da atmosfera, em uma região delimitada da superfície terrestre. Estudamse os fatores climáticos, para que, através deles, possam ser inferidas hipóteses de causalidade quanto a algum fator de risco cuja variação na natureza dependa da variação de algum fator climático.

Conforme Ayoade (2010), algumas doenças tendem a ser predominantes em certas zonas climáticas, enquanto outras, particularmente as infectocontagiosas, tendem a seguir um padrão sazonal em sua incidência.

As variáveis, precipitação, temperatura e umidade relativa do ar foram selecionadas para análise, pois são elementos instáveis da atmosfera e segundo Ayoade (2010) a influência na saúde humana pode ser direta, indireta, positiva ou negativa. No entanto, para estudar saúde é necessário analisar, a partir de outras perspectivas, o contexto no qual o indivíduo está inserido. Verificando a relação do mesmo com a natureza, as condições básicas de moradia, educação e saúde, que de forma direta possuem relação com os agravos à saúde. Estudos nacionais têm verificado associações positivas entre as condições climáticas e doenças respiratórias (MOURA et al., 2008; CASTRO et al., 2009; SOUSA et al., 2012). No âmbito internacional também encontramos alguns esforços (SALVI, 2007; XU et al., 2011).

No Brasil, existem vários estudos correlacionando a influência do meio ambiente sobre o organismo humano (NATALI et. al., 2011; SOUZA et al., 2014; PONTES et al., 2016), tentando compreender o complexo processo de saúde e doença que avaliaram os impactos das condições do clima e tempo sobre a saúde da população. A seguir são apresentados alguns desses estudos que abordaram essas variáveis ambientais e os efeitos adversos na saúde da população.

Gouveia et al., (2003) realizaram um estudo no qual analisaram esses impactos nas duas maiores cidades brasileiras, Rio de Janeiro (RJ) e São Paulo (SP). Nascimento et al., (2006) estimaram em São José dos Campos (SP) a associação das internações por pneumonias com o aumento dos poluentes atmosféricos. $\mathrm{O}$ estudo confirma que o potencial deletério dos poluentes do ar sobre a saúde pode ser detectado, também, em cidades de médio porte. A magnitude do efeito foi semelhante ao observado na cidade de São Paulo. Além disso, mostra a elevada susceptibilidade das crianças aos efeitos adversos advindos da exposição aos contaminantes atmosféricos.

Natali et al. (2011), descreveram as principais características de distribuição temporal, por faixa etária e por causa específica, da morbidade hospitalar por doenças respiratórias na infância e adolescência no Município de São Paulo, $\mathrm{SP}$, a partir dos registros de internações em hospitais do Sistema Único de Saúde (SUS), no período de 2000 a 2004. Constataram que as internações por doenças respiratórias

(pneumonia, broncopneumonia e asma) de crianças e adolescentes apresentam padrão de distribuição dependente da faixa etária e da sazonalidade. Quanto menor a faixa etária maior o número de internações. 
Alves et al. (2015), por sua vez, avaliaram os efeitos das variáveis climáticas (precipitação pluvial, temperatura média do ar e umidade relativa do ar) na incidência de doenças relacionadas à infecções das vias aéreas superiores (IVAS) no município de Monteiro - PB. Os resultados evidenciaram que os valores máximos do número de registros de IVAS ocorrem no período do outono-inverno (março a agosto) e mínimos no período da primavera-verão (setembro a fevereiro).

Azevedo et al (2015), avaliaram a influência das variáveis climáticas (precipitação pluvial, temperatura e umidade do ar) na incidência de infecções respiratórias agudas (IRA) em crianças menores de dois anos nos municípios de Monteiro e Campina Grande, na Paraíba, no período de 1998 a 2012.

Pontes et al (2016), avaliaram as doenças respiratórias em relação a algumas variáveis climáticas (temperatura do ar, precipitação pluviométrica e umidade relativa do ar) no município de Ponta Grossa, PR, no período de janeiro de 1998 a dezembro de 2013. Foi possível observar uma grande relevância da pneumonia nas taxas de internações. Dentre essas variável climática a que se mostrou mais correlacionada à pneumonia foi a temperatura mínima com elevada significância estatística.

Miranda (2016) investigou a associação dos elementos meteorológicos (umidade relativa mínima e temperaturas máxima e mínima diárias) com as internações por morbidades respiratórias (pneumonia e gripe) no município de São Paulo, SP. Observaram certa tendência do aumento das internações quando há aumento das temperaturas máximas e mínimas, e queda da umidade relativa mínima em até sete dias antes dos picos de internação.

Azevedo et al. (2017), avaliaram os efeitos das variações sazonais do clima na ocorrência de internações por doenças respiratórias por Influenza e Pneumonia na população idosa da Região Metropolitana de João Pessoa no Estado da Paraíba. Verificou-se que os maiores picos de internações por pneumonia ocorrem no outono e inverno. Portanto, sugerindo uma associação entre o frio e as internações por pneumonia.

Mandú et al. (2019) avaliaram e compararam o efeito da temperatura e umidade relativa do ar na ocorrência de doenças respiratórias nas seguintes cidades brasileiras: Fortaleza, Manaus, Natal e Palmas. Para isso, foram utilizados dados mensais de temperatura e umidade relativa do ar registrados por estações meteorológicas convencionais alocadas nas capitais acima mencionadas, disponibilizados pelo Banco de Dados Meteorológicos para Ensino e Pesquisa (BDMEP). Os resultados apontam associação significativa inversa em Fortaleza e Natal, e direta em Manaus e Palmas com a temperatura média do ar, e para a umidade relativa do ar, já que mostraram associação.

No âmbito internacional também são evidenciadas várias experiências que procuraram estudar a associação entre saúde e clima. Weber et al. (1988), efetuaram um estudo na Gâmbia, no período de 1993 a 1996, em crianças menores de dois anos internadas com infecções respiratórias. Os autores concluíram que as internações ocorreram com maior frequência nos meses de julho a novembro durante o período chuvoso.

Strachan e Sanders (1989) avaliaram a habitação úmida e asma infantil e os efeitos respiratórios da temperatura do ar interior e da umidade relativa. Os resultados encontrados foram contrários à crença generalizada de que a temperatura interna e a umidade são determinantes importantes da saúde respiratória, embora não excluam diretamente os efeitos causados por ácaros ou moldes, cuja sobrevivência é determinada pela umidade de seus respectivos microambientes. 
Chan et al. (1999), realizaram um estudo em Hong Kong, no período de 1993 a 1997, em 9635 crianças hospitalizadas com infecções respiratórias agudas. Os autores concluíram que os picos de incidência das infecções ocorriam no período de abril a setembro e estavam relacionados com as chuvas, as baixas temperaturas e a elevada umidade relativa do ar.

Oluleye e Akinbobola (2010) estudaram a relação entre infecção por pneumonia, precipitação e temperatura do ar em Lagos, Nigéria. Detectaram que a mudança climática significativa foi detectada tanto na precipitação quanto na temperatura do ar. Concluíram que os parâmetros climáticos, as chuvas e a temperatura do ar têm uma profunda influência na ocorrência de pneumonia e são responsáveis diretamente pelo aumento intratável das doenças. $\mathrm{Xu}$ et al. (2011), investigaram as características epidemiológicas do Mycoplasma Pneumoniae (MPP) na infância e observaram se existe uma relação entre características epidemiológicas e fatores meteorológicos em Hangzhou na China. O estudo mostrou que a taxa de MPP foi maior em crianças mais velhas do que nas mais jovens. As meninas tiveram uma taxa positiva maior de MPP do que os meninos. Em Hangzhou, o MPP foi mais prevalente no verão e no outono. A temperatura do ar foi o único fator meteorológico que afetou a prevalência do MPP.

$\mathrm{Xu}$ et al. (2014), examinaram o impacto da temperatura na pneumonia infantil em Brisbane, Austrália. Identificaram que as crianças entre 2-5 anos e as crianças do sexo feminino eram particularmente vulneráveis aos impactos do calor e do frio, e as crianças indígenas eram sensíveis ao calor. As ondas de calor e de frio tiveram efeitos agregados significativos na pneumonia infantil e a magnitude desses efeitos aumentou com intensidade e duração. Houve mudanças ao longo do tempo nos efeitos principais e adicionados de temperatura na pneumonia infantil. As crianças, especialmente aquelas femininas e indígenas, devem ser particularmente protegidas contra temperaturas extremas. Concluíram que o desenvolvimento futuro de sistemas de alerta precoce deve levar em consideração a mudança ao longo do tempo no impacto da temperatura na saúde infantil.

Chen et al. (2014), utilizaram as variáveis meteorológicas precipitação, temperatura e umidade relativa na ocorrência de doença dos legionários (LD) em Taiwan, no período de 1995-2011. A doença dos legionários (DL) é uma forma aguda de pneumonia, e a mudança climática é considerada um fator de risco plausível. E é provável que um aumento da precipitação diária seja um fator climático crítico que desencadeie a ocorrência desse tipo de doença, em que o risco é particularmente significativo. Além disso, as análises estratificadas mostraram ainda que associações positivas de precipitação com incidência de LD foram apenas significativas em grupos masculinos e idosos e durante a estação quente.

Qiu et al, (2016), analisaram a associação entre temperatura ambiente e pneumonia em idoso e avaliaram a carga de doença atribuível a temperaturas frias e quentes em Hong Kong, China. Foram realizadas análises de subgrupos para examinar a diferença no gênero. Concluíram que a maior parte da carga relacionada à temperatura para internações por pneumonia em Hong Kong era atribuível a temperaturas frias, e os homens idosos apresentaram maior susceptibilidade.

Como se observa, existem vários estudos que analisaram o efeito das variáveis climáticas (temperatura do ar, umidade relativa do ar e precipitação) sobre as doenças respiratórias denotando a relevância de realizar a presente pesquisa. Face ao exposto, entende-se que avaliar os efeitos dessas variáveis climáticas na incidência dos casos de pneumonia na cidade de Campina Grande, PB se apresenta 
como um gap que pode contribuir para a gestão da saúde local e a elaboração de políticas públicas de saúde.

\section{MATERIAL E MÉTODOS}

\section{Área e período do estudo}

O estudo foi realizado no Município de Campina Grande, localizado no Estado da Paraíba, durante a série histórica de janeiro de 1998 a dezembro de 2016, correspondendo a 19 anos.
O município de Campina Grande (Figura 1) situa-se no agreste paraibano. Possui um clima tropical com temperaturas moderadas. Chove muito mais no verão do que no inverno. A classificação do clima segundo a Köppen e Geiger tem uma temperatura média em torno de $22.9^{\circ} \mathrm{C}$, podendo atingir $32^{\circ} \mathrm{C}$ nos dias mais quentes, 15 nas noites mais frias do ano. A pluviosidade média anual é $765 \mathrm{~mm}$. A umidade relativa do ar está entre 75 a $83 \%$. $\mathrm{O}$ período chuvoso começa em abril e termina em agosto (TRAVASSOS, 2012).

Figura 1 - Localização do Município de Campina Grande, PB.

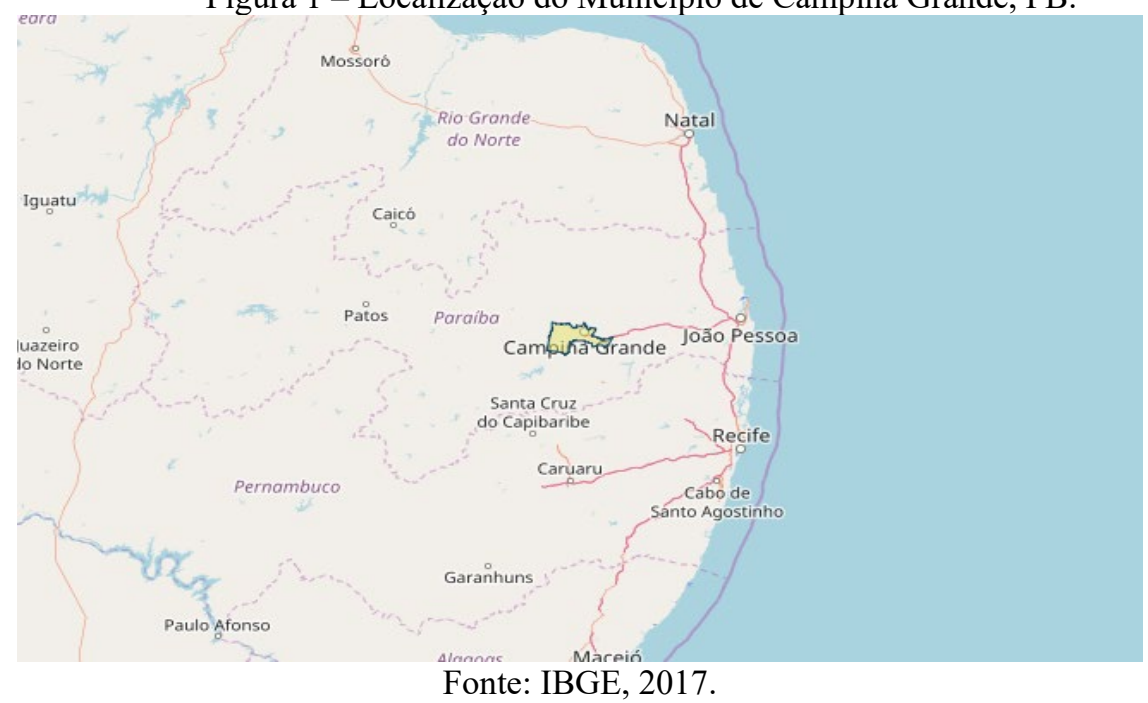

\section{Tipo de Estudo e Tratamento Estatístico}

Foi realizado um estudo epidemiológico, retrospectivo, com característica descritiva, de corte transversal a fim de identificar e analisar uma possível relação entre casos de pneumonia e as variáveis meteorológicas (precipitação pluviométrica, temperatura do ar e umidade relativa do ar) no contexto do município de Campina Grande, Paraíba.

Quanto à natureza, classifica-se como quantitativa, visto que foram analisados os casos de internação hospitalar por pneumonia e as possíveis relações destas com as variáveis da pesquisa.

O tratamento estatístico foi composto pelas medidas de associação, $\boldsymbol{r}$ de Pearson, para verificar a correlação entre os casos de pneumonia com as variáveis ambientais (temperaturas, umidade relativa do ar e precipitação) na respectiva região do estudo. Para realização do teste de Correlação de Pearson foram consideradas variáveis independentes, as variáveis meteorológicas e, como variáveis dependentes, pneumonia. A variável dependente é aquilo que acontece durante uma investigação na mensuração da condição de saúde-doença (desfecho) e a variável independente é o fator que precede o desfecho (exposição), conforme defendem Callegari-Jacques (2009) e Franco (2017). 


\section{RESULTADOS E DISCUSSÃO}

A seguir são apresentados os resultados encontrados ao realizar o cruzamento entre as variáveis meteorológicas e a faixa etária dos casos de pneumonia em Campina Grande, durante os anos de 1998 a 2016.
Correlação dos casos de pneumonia com as variáveis meteorológicas por faixa etária em Campina Grande, PB

$\mathrm{Na}$ Tabela 1 estão dispostos os valores dos coeficientes de correlação de Pearson (r) e de Determinação $\left(\mathrm{R}^{2}\right)$ encontrados entre variável meteorológica precipitação e o número de casos de pneumonia, ocorridos em Campina Grande nos anos de 1998 a 2016.

Tabela 1 - Correlação entre os casos de pneumonia e precipitação em Campina Grande ${ }^{\text {iv }}$

\begin{tabular}{|c|c|c|c|c|c|c|c|c|c|c|c|c|}
\hline \multirow{2}{*}{ Anos } & \multicolumn{3}{|c|}{$<5$ anos } & \multicolumn{3}{|c|}{ Entre 5 e 19 anos } & \multicolumn{3}{|c|}{ Entre 20 e 59 anos } & \multicolumn{3}{|c|}{ Acima de 60 anos } \\
\hline & $r$ & $\mathrm{R}^{2}$ & Clas & $r$ & $\overline{\mathrm{R}^{2}}$ & Classif. & $r$ & $\overline{\mathrm{R}^{2}}$ & Classif. & $r$ & $\mathrm{R}^{2}$ & Classif. \\
\hline 1998 & 0,35 & 0,12 & Média & 0,39 & 0,16 & Média & 0,55 & 0,30 & Média & 0,70 & 0,50 & Forte \\
\hline 1999 & 0,02 & 0,00 & Nula & $-0,16$ & 0,03 & Fraca & 0,11 & 0,01 & Fraca & $-0,01$ & 0,00 & Nula \\
\hline 2000 & 0,44 & 0,19 & Média & 0,01 & 0,00 & Nula & 0,36 & 0,13 & Média & $-0,28$ & 0,08 & Fraca \\
\hline 2001 & $-0,03$ & 0,00 & la & $-0,44$ & 0,19 & Média & $-0,64$ & 0,41 & rte & $-0,63$ &, 40 & Forte \\
\hline 2002 & 0,85 & 0,71 & Forte & 0,31 & 0,10 & Média & 0,30 & 0,09 & Fraca & 0,21 & 0,05 & Fraca \\
\hline 2003 & 0,23 & 0,05 & $\mathrm{ca}$ & $-0,20$ & 0,04 & $\mathrm{ca}$ & $-0,13$ & 0,02 & $\mathrm{ca}$ & 0,00 & 0,00 & Nula \\
\hline 2004 & $-0,45$ & 0,20 & Média & $-0,90$ & 0,81 & Fortís. & $-0,56$ & 0,32 & Média & $-0,24$ & 0,06 & Fraca \\
\hline 2005 & $-0,11$ & 0,01 & Fraca & $-0,56$ & 0,31 & Média & 0,02 & 0,00 & Nula & $-0,05$ & 0,00 & Nula \\
\hline 2006 & 0,46 & 0,21 & Média & 0,18 & 0,03 & Fraca & 0,02 & 0,00 & Nula & $-0,14$ & 0,02 & Fraca \\
\hline 2007 & 0,37 & 0,13 & Média & $-0,04$ & 0,00 & Nula & 0,09 & 0,01 & Fraca & 0,32 & 0,10 & Média \\
\hline 2008 & $-0,21$ & 0,04 & Fraca & $-0,21$ & 0,05 & Fraca & $-0,05$ & 0,00 & Nula & 0,20 & 0,04 & Fraca \\
\hline 2009 & 0,03 & 0,00 & Nula & $-0,44$ & 0,19 & Média & $-0,39$ & 0,15 & Média & $-0,29$ & 0,08 & Fraca \\
\hline 2010 & 0,51 & 0,26 & Média & $-0,40$ & 0,16 & Média & $-0,30$ & 0,09 & Fraca & $-0,11$ & 0,01 & Fraca \\
\hline 2011 & 0,55 & 0,30 & Média & $-0,36$ & 0,13 & Média & 0,18 & 0,03 & $\mathrm{ca}$ & 0,09 & 0,01 & Fraca \\
\hline 2012 & 0,11 & 0,01 & Fraca & 0,24 & 0,06 & Fraca & 0,31 & 0,09 & Fraca & 0,44 & 0,20 & Média \\
\hline 2013 & 0,53 & 0,28 & Média & 0,35 & 0,12 & Média & 0,42 & 0,18 & Média & 0,46 & 0,21 & Média \\
\hline 2014 & 0,62 & 0,39 & Forte & $-0,22$ & 0,05 & Fraca & 0,27 & 0,08 & Fraca & 0,11 & 0,01 & Fraca \\
\hline 2015 & 0,62 & 0,39 & Forte & $-0,22$ & 0,05 & Fraca & 0,27 & 0,08 & Fraca & 0,11 & 0,01 & Fraca \\
\hline 2016 & 0,15 & 0,02 & Fraca & $-0,11$ & 0,01 & Fraca & 0,44 & 0,20 & Média & $-0,29$ & 0,08 & Fraca \\
\hline
\end{tabular}

Fonte: Dados da pesquisa, 2017.

A faixa etária em menores de 5 anos apontou que durante os anos de 2002 $\left(\mathrm{r}=0,85\right.$ e $\left.\mathrm{R}^{2}=0,71\right), \quad 2014 \quad(\mathrm{r}=0,62 \quad \mathrm{e}$ $\left.\mathrm{R}^{2}=0,39\right)$ e $2015\left(\mathrm{r}=0,62\right.$ e $\left.\mathrm{R}^{2}=0,39\right)$ houve uma associação entre forte e média no tocante à precipitação e o número de casos de pneumonia. Os anos de $1998(\mathrm{r}=0,35$ e $\left.\mathrm{R}^{2}=0,12\right), 2000\left(\mathrm{r}=0,44\right.$ e $\left.\mathrm{R}^{2}=0,19\right), 2004$ $\left(\mathrm{r}=-0,45 \quad\right.$ e $\left.\quad \mathrm{R}^{2}=0,20\right), \quad 2006 \quad(\mathrm{r}=0,46 \quad$ e $\left.\mathrm{R}^{2}=0,21\right), 2007\left(\mathrm{r}=0,37\right.$ e $\left.\mathrm{R}^{2}=0,13\right), 2010$ $\left(\mathrm{r}=0,51\right.$ e $\left.\mathrm{R}^{2}=0,26\right), 2011\left(\mathrm{r}=0,55 \mathrm{R}^{2}=0,30\right)$, $2013\left(\mathrm{r}=0,53\right.$ e $\left.\mathrm{R}^{2}=0,28\right)$, com exceção do ano de $2004\left(\mathrm{r}=-0,45\right.$ e $\left.\mathrm{R}^{2}=0,20\right)$ que apresentou um comportamento de correlação negativa média, ou seja, quando há um aumento dos seus respectivos valores, há uma redução na taxa de internação pela doença.
Observe que os anos de 1999, 2001, 2003, 2005, 2008, 2009, 2012 e 2016 apresentaram uma correlação entre fraca e nula para a associação da precipitação com os casos de pneumonia nessa faixa etária. Em praticamente mais da metade dos anos estudados houve correlação entre média e forte, reforçando aqui a tese que a precipitação exerce influência sobre os casos de pneumonia dentro dessa faixa etária. Existem casos de asma nas outras faixas etárias analisadas, logo a que mais prevalece é a faixa menor que 5 anos.

Para a faixa etária entre 5 e 19 anos o ano de $2004 \quad\left(\mathrm{r}=-0,90\right.$ e $\left.\mathrm{R}^{2}=0,81\right)$ apresentou uma correlação negativa forte, demonstrando que $81 \%$ dos casos de pneumonia naquele ano podem ter sofrido influência da variável precipitação. Os anos 
que apresentaram uma correlação média foram $1998 \quad\left(\mathrm{r}=0,39\right.$ e $\left.\mathrm{R}^{2}=0,16\right), 2002$ $\left(\mathrm{r}=0,31 \quad\right.$ e $\left.\mathrm{R}^{2}=0,10\right), \quad 2013 \quad(\mathrm{r}=0,35 \quad \mathrm{e}$ $\left.\mathrm{R}^{2}=0,12\right)$. Os anos de $2001(\mathrm{r}=-0,44 \mathrm{e}$ $\left.\mathrm{R}^{2}=0,19\right), 2005\left(\mathrm{r}=-0,56\right.$ e $\left.\mathrm{R}^{2}=0,31\right), 2009$ $\left(\mathrm{r}=-0,44\right.$ e $\left.\mathrm{R}^{2}=0,19\right), 2010 \quad(\mathrm{r}=-0,40$ e $\left.\mathrm{R}^{2}=0,16\right), 2011 \quad\left(\mathrm{r}=-0,36\right.$ e $\left.\mathrm{R}^{2}=0,13\right)$, apresentaram uma correlação negativa média. Os anos de 1999, 2000, 2003, 2006, 2007, 2008, 2012, 2014, 2015 e 2016 apresentaram uma correlação entre fraca e nula. Na faixa etária acima de 60 anos de idade há uma correlação entre nula e fraca bastante acentuada durante 14 anos mostrando que a explicação para esses casos é improvável.

Ao analisar os dados da Tabela 2 é possível observar que os coeficientes de correlação e determinação apontam, no decorrer dos anos, os casos de pneumonia que apresentam maior correlação inversa com a temperatura do ar, confirmação negativa nos coeficientes de correlação e nos coeficientes de determinação. Houve uma forte correlação inversa em nove anos e média em oito anos, retratando a influência que essa variável meteorológica exerce nes casos específicos. Portanto, quanto menor a temperatura nessa localidade maiores são as possibilidades de apresentar número maior de casos de pneumonia. Observa-se, novamente, a faixa etária menor que cinco anos prevalece em relação as outras estudadas.

Veja que a faixa entre 5 e 19 anos apresenta correlação inversa forte em apenas três anos: $1998\left(\mathrm{r}=-0,76\right.$ e $\left.\mathrm{R}^{2}=0,57\right)$, $2001\left(\mathrm{r}=0,78\right.$ e $\left.\mathrm{R}^{2}=0,61\right), 2008(\mathrm{r}=-0,64$ e $\left.\mathrm{R}^{2}=0,41\right)$ e $2013\left(\mathrm{r}=-0,78\right.$ e $\left.\mathrm{R}^{2}=0,61\right)$. Na faixa etária entre 20 e 59 anos houve apenas três correlações fortes, sendo duas inversas (anos de 1998 com $\mathrm{r}=-0,78$ e $\mathrm{R}^{2}=0,60$ e ano
2012 com $\mathrm{r}=-0,67$ e $\left.\mathrm{R}^{2}=0,44\right)$ e uma direta (ano de $2001 \mathrm{r}=0,74$ e $\mathrm{R}^{2}=0,54$ ). Resultado semelhante foi obtido para a faixa etária acima de 60 anos, que perfaz um total de 10 anos com correlações classificadas entre média e forte e 9 anos entre nula e fraca.

Ayoade (2010) refere que "algumas doenças tendem a ser preferenciais em certas zonas climáticas, enquanto algumas outras, principalmente as contagiosas, tendem a seguir um padrão sazonal". Porém, Martins e Trevisol (2013) enfocam que é importante ressaltar que, embora se perceba que surtos de infecções respiratórias estejam associados a baixas temperaturas, elas não são a causa das infecções, elas apenas influenciam as interações entre patógeno e hospedeiro, aumentando as chances de contaminação.

Os achados do estudo se alinham aos que foram encontrados por Carneseca et al. (2010) e Santos et al. (2016), em que evidenciaram que o número de internações por pneumonia em crianças com até 10 anos é influenciado pelo aumento na temperatura. Com relação ao número de internações de idosos com mais de 75 anos, devido a essa mesma causa, o estudo também evidenciou que ele aumenta no período do outono e diminui nos finais de semana ou quando a umidade mínima diária do ar aumenta.

Também corroboram a afirmação de Brasil (2010) de que o maior número de internações por pneumonias ocorre nas idades extremas, para crianças e idosos acima de 60 anos. Isso ocorre devido ao sistema imunológico frágil de pessoas nessas idades. Nos idosos, isso pode ser agravado pela presença de outras doenças comuns na terceira idade como diabetes, problemas cardíacos, renais, entre outras. 
Tabela 2 - Correlação entre os casos de pneumonia e temperatura do ar em Campina Grande

\begin{tabular}{|c|c|c|c|c|c|c|c|c|c|c|c|c|}
\hline \multirow{2}{*}{ Anos } & \multicolumn{3}{|c|}{$<5$ anos } & \multicolumn{3}{|c|}{ Entre 5 e 19 anos } & \multicolumn{3}{|c|}{ Entre 20 e 59 anos } & \multicolumn{3}{|c|}{ Acima de 60 anos } \\
\hline & $r$ & $\mathrm{R}^{2}$ & 2 & $r$ & $\mathrm{R}^{2}$ & Classif. & $r$ & $\mathrm{R}^{2}$ & Classif. & $r$ & $\mathrm{R}^{2}$ & Classif. \\
\hline 1998 & $-0,51$ & $\overline{0,2}$ & & 0,76 & 57 & & $80_{7}$ & 0,60 & & 0,87 & 0,76 & \\
\hline 1999 & $-0,40$ & 0,16 & & $-0,40$ & 0,16 & & $-0,57$ & 0,32 & & $-0,54$ & 0,29 & \\
\hline 2000 & $-0,51$ & 0,26 & Me & $-0,24$ & 0,06 & $\mathrm{Fr}$ & $-0,46$ & 0,22 & $\mathrm{ia}$ & 0,18 & 0,03 & \\
\hline 2001 & $-0,63$ & 0,39 & & 0,78 & 0,61 & & 0,74 & 0,54 & & 0,31 & 0,09 & \\
\hline 2002 & 0,65 & 0,42 & & 0,22 & 0,05 & & 0,39 & 0,15 & & 0,34 & 0,12 & \\
\hline 2003 & $-0,7$ & 0,52 & & 0,17 & 0,03 & & 37 & 0,14 & & 51 & 0,26 & \\
\hline 2004 & $-0,13$ & 0,02 & $\mathrm{ca}$ & 0,44 & 0,19 & Média & $-0,06$ & 0,00 & Nula & $-0,07$ & 0,00 & Nula \\
\hline 2005 & $-0,50$ & 0,25 & M & 0,36 & 0,13 & $\overline{\text { Mé }}$ & 0,06 & 0,00 & $\bar{N}$ & $-0,28$ & 0,08 & $\mathrm{ca}$ \\
\hline 2006 & $-0,7$ & 0,56 & & $-0,48$ & 0,23 & & $-0,25$ & 0,06 & & $-0,48$ & 0,23 & \\
\hline 2007 & \begin{tabular}{|l|}
$-0,64$ \\
\end{tabular} & 0,40 & & $-0,28$ & 0,08 & & $-0,11$ & 0,01 & & $-0,67$ & 0,45 & \\
\hline 2008 & $-0,70$ & 0,48 & Forte & $-0,64$ & 0,41 & Forte & 0,02 & 0,00 & & 0,20 & 0,04 & $\mathrm{ca}$ \\
\hline 2009 & \begin{tabular}{|l|}
$-0,59$ \\
\end{tabular} & 0,35 & lia & $-0,21$ & 0,04 & $\mathrm{ca}$ & $-0,03$ & 0,00 & Nula & $-0,24$ & 0,06 & Fraca \\
\hline 2010 & \begin{tabular}{|l|}
$-0,53$ \\
\end{tabular} & 0,29 & Média & $-0,30$ & 0,09 & Fraca & 0,29 & 0,08 & & 0,04 & 0,00 & Nula \\
\hline 2011 & \begin{tabular}{|l|}
$-0,42$ \\
\end{tabular} & 0,18 & & 0,40 & 0,16 & Mé & $-0,34$ & 0,12 & & $-0,57$ & 0,33 & Média \\
\hline 2012 & $-0,30$ & 0,09 & $\mathrm{ca}$ & 0,09 & 0,01 & Fraca & $-0,67$ & 0,44 & Forte & $-0,23$ & 0,05 & Fraca \\
\hline 2013 & $-0,49$ & 0,24 & Média & $-0,78$ & 0,61 & Forte & $-0,19$ & 0,04 & Fraca & $-0,67$ & 0,45 & Forte \\
\hline 2014 & $-0,69$ & 0,48 & & 0,05 & 0,00 & & $-0,37$ & 0,13 & & $-0,36$ & 0,13 & Média \\
\hline 2015 & $-0,69$ & 0,48 & Forte & 0,05 & 0,00 & Nula & $-0,37$ & 0,13 & Média & $-0,36$ & 0,13 & Média \\
\hline 2016 & \begin{tabular}{|l|}
$-0,77$ \\
\end{tabular} & 0,60 & Forte & $-0,47$ & 0,22 & Média & $-0,30$ & 0,09 & $\mathrm{Fr}$ & $-0,10$ & 0,01 & Fraca \\
\hline
\end{tabular}

Fonte: Dados da pesquisa, 2017.

A Tabela 3 contém os coeficientes de correlação (r) e os coeficientes de determinação $\left(\mathrm{R}^{2}\right)$ do elemento meteorológico umidade relativa do ar com a pneumonia no período de 1999 a 2009. Observa-se uma forte correlação positiva em sete anos com valores do $r$ oscilando entre 0,62 e 0,82 dentro da faixa etária menor que 5 anos (faixa de maior predominância de correlação). Veja que no ano 2000 o $\mathrm{R}^{2}=0,53$, em 2002 o $\mathrm{R}^{2}=0,68$, 2006 com $\mathrm{R}^{2}=0,49,2007$ fica com $\mathrm{R}^{2}=0,38$, $2013 \mathrm{R}^{2}=0,52,2014$ e $2015 \mathrm{R}^{2}=0,41$. Isso sugere que essas variáveis meteorológicas estão bem correlacionadas com a doença (diretamente proporcionais). Enquanto nas outras faixas etárias houve distribuição de correlações em maior quantidade, classificadas entre média, nula e fraca.

Essa constatação se alinha ao que foi encontrado no estudo de Pontes et al.
(2016), quando avaliaram as doenças do aparelho respiratório em relação a algumas variáveis climáticas (temperatura do ar, precipitação pluviométrica e umidade relativa do ar) no município de Ponta Grossa -PR. Verificaram que no verão há um índice mais baixo de internações por pneumonia com um aumento significativo durante o outono e seu pico máximo sendo claramente observado nos meses de inverno. Tal fato evidencia que durante o período de baixas temperaturas e elevada umidade do ar há um aumento das taxas de internações decaindo novamente na primavera, com a retomada lenta da elevação da temperatura do ar, reforçando os resultados encontrados nesta pesquisa. 
Tabela 3 - Correlação entre os casos de pneumonia e umidade relativa do ar em Campina Grande

\begin{tabular}{|c|c|c|c|c|c|c|c|c|c|c|c|c|}
\hline \multirow{2}{*}{ Anos } & \multicolumn{3}{|c|}{$<5$ anos } & \multicolumn{3}{|c|}{ Entre 5 e 19 anos } & \multicolumn{3}{|c|}{ Entre 20 e 59 anos } & \multicolumn{3}{|c|}{ Acima de 60 anos } \\
\hline & $\boldsymbol{r}$ & $\overline{\mathrm{R}^{2}}$ & Classif. & $\boldsymbol{r}$ & $\overline{\mathrm{R}^{2}}$ & Classif. & $\boldsymbol{r}$ & $\overline{\mathrm{R}^{2}}$ & Classif. & $\boldsymbol{r}$ & $\overline{\mathrm{R}^{2}}$ & Classif. \\
\hline 1998 & 0,17 & 0,03 & Fraca & 0,45 & 0,20 & Média & 0,58 & 0,34 & Média & 0,75 & 0,57 & Forte \\
\hline 1999 & 0,36 & 0,13 & Média & 0,09 & 0,01 & Fraca & 0,45 & 0,20 & Média & 0,27 & 0,07 & Fraca \\
\hline 2000 & 0,73 & 0,53 & Forte & 0,56 & 0,32 & Média & 0,74 & 0,55 & Forte & $-0,23$ & 0,05 & Fraca \\
\hline 2001 & 0,21 & 0,04 & Fraca & $-0,70$ & 0,49 & Forte & $-0,67$ & 0,45 & Forte & $-0,61$ & 0,37 & Forte \\
\hline 2002 & 0,82 & 0,68 & Forte & $-0,04$ & 0,00 & Nula & $-0,09$ & 0,01 & Fraca & $-0,17$ & 0,03 & Fraca \\
\hline 2003 & 0,46 & 0,21 & Média & $-0,27$ & 0,07 & Fraca & $-0,01$ & 0,00 & Nula & 0,09 & 0,01 & Fraca \\
\hline 2004 & 0,12 & 0,02 & Fraca & $-0,63$ & 0,40 & Forte & $-0,16$ & 0,03 & Fraca & 0,09 & 0,01 & Fraca \\
\hline 2005 & $-0,22$ & 0,05 & Fraca & $-0,42$ & 0,17 & Média & 0,05 & 0,00 & Nula & $-0,11$ & 0,01 & Fraca \\
\hline 2006 & 0,70 & 0,49 & Forte & 0,35 & 0,12 & Média & $-0,06$ & 0,00 & Nula & 0,10 & 0,01 & Fraca \\
\hline 2007 & 0,62 & 0,38 & Forte & 0,09 & 0,01 & Fraca & 0,13 & 0,02 & Fraca & 0,77 & 0,60 & Forte \\
\hline 2008 & 0,10 & 0,01 & Fraca & 0,09 & 0,01 & Fraca & $-0,40$ & 0,16 & Média & $-0,38$ & 0,15 & Média \\
\hline 2009 & 0,39 & 0,15 & Média & $-0,14$ & 0,02 & Fraca & $-0,46$ & 0,21 & Média & $-0,24$ & 0,06 & Fraca \\
\hline 2010 & 0,42 & 0,18 & Média & $-0,20$ & 0,04 & Fraca & $-0,52$ & 0,27 & Média & $-0,20$ & 0,04 & Fraca \\
\hline 2011 & 0,58 & 0,33 & Média & $-0,58$ & 0,34 & Média & 0,40 & 0,16 & Média & 0,45 & 0,20 & Média \\
\hline 2012 & 0,15 & 0,02 & Fraca & $-0,07$ & 0,01 & Fraca & 0,42 & 0,18 & Média & 0,23 & 0,05 & Fraca \\
\hline 2013 & 0,72 & 0,52 & Forte & 0,73 & 0,54 & Forte & 0,15 & 0,02 & Fraca & 0,54 & 0,29 & Média \\
\hline 2014 & 0,64 & 0,41 & Forte & $-0,04$ & 0,00 & Nula & 0,22 & 0,05 & Fraca & 0,07 & 0,00 & Nula \\
\hline 2015 & 0,64 & 0,41 & Forte & $-0,04$ & 0,00 & Nula & 0,22 & 0,05 & Fraca & 0,07 & 0,00 & Nula \\
\hline 2016 & 0,48 & 0,23 & Média & 0,26 & 0,07 & Fraca & 0,25 & 0,06 & Fraca & $-0,34$ & 0,11 & Média \\
\hline
\end{tabular}

Fonte: Dados da pesquisa, 2017.

Correlação dos casos de pneumonia e as variáveis meteorológicas em Campina Grande, PB

Analisando a Tabela 4, verifica-se que é a partir do mês de maio até o mês de novembro que geralmente ocorre uma alta nos casos de internação hospitalar por doenças respiratórias no município de Campina Grande.

Os casos de pneumonia apresentam uma quantidade maior nos meses de maio, junho, julho e agosto. É possível observar que nesses meses de maior precipitação ocorreram menores temperaturas do ar e as maiores umidades relativas do ar. Esses meses fazem referência a períodos chuvosos na cidade de Campina Grande, período em que a umidade do ar tende a ser muito alta, o que de fato ocorreu na cidade, contribuindo dessa forma para a formação de mofos, fungos e ácaros, ou seja, vetores que ampliam as chances de potencializar número de pessoas acometidas com doenças respiratórias.

Tabela 4 - Distribuição mensal dos casos de pneumonia e variáveis meteorológicas

\begin{tabular}{c|c|c|c|c}
\hline Meses & $\begin{array}{c}\text { Precipitação } \\
\text { pluviométrica }\end{array}$ & $\begin{array}{c}\text { Temperatura do } \\
\text { ar }\end{array}$ & $\begin{array}{c}\text { Umidade } \\
\text { Relativa do } \\
\text { Ar }\end{array}$ & $\begin{array}{c}\text { Total de casos } \\
\text { de Pneumonia }\end{array}$ \\
\hline Jan & 1074,4 & 30,5 & 75,5 & 3788 \\
\hline Fev & 1323,6 & 30,5 & 76,3 & 3122 \\
\hline Mar & 1739,3 & 30,3 & 77,5 & 3667 \\
\hline Abr & 1529,1 & 29,7 & 78,4 & 4363 \\
\hline Mai & $\mathbf{1 9 5 9}$ & $\mathbf{2 8 , 1}$ & $\mathbf{8 1 , 3}$ & $\mathbf{5 1 0 4}$ \\
\hline Jun & $\mathbf{2 5 8 9 , 8}$ & $\mathbf{2 6 , 3}$ & $\mathbf{8 3 , 7}$ & $\mathbf{5 6 2 3}$ \\
\hline Jul & $\mathbf{2 1 9 7 , 4}$ & $\mathbf{2 5 , 7}$ & $\mathbf{8 3 , 4}$ & $\mathbf{5 6 0 9}$ \\
\hline Ago & $\mathbf{1 4 2 7 , 5}$ & $\mathbf{2 6 , 8}$ & $\mathbf{8 0 , 5}$ & $\mathbf{4 7 6 5}$ \\
\hline Set & 636,3 & 28,2 & 76,0 & 4477 \\
\hline Out & 275,9 & 29,6 & 72,9 & 4543 \\
\hline Nov & 193,4 & 30,6 & 71,8 & 4594 \\
\hline Dez & 288,6 & 30,8 & 73,1 & 4119 \\
\hline
\end{tabular}

Fonte: Dados da pesquisa, 2017. 
Os meses com menores registros de casos de pneumonia foram janeiro (3.788) fevereiro (3.122) e março (3.667), correspondendo ao período de verão. Verifica-se que no período chuvoso (maio a agosto) foram registrados 21.101 casos de pneumonia, o que corresponde $39,24 \%$ dos casos.

Com relação às variáveis climáticas, observa-se que a estação chuvosa compreende os meses de maio, junho, julho e agosto em Campina Grande. Já com relação à umidade relativa do ar, o município apresenta valores mais elevados nesse período, o que explica o aumento no número de internações. Observa-se que as variáveis temperatura e umidade relativa do ar apresentam comportamentos que acompanham as estações do ano, com temperaturas mais elevadas na primavera/verão e umidade relativa do ar mais baixa no outono/inverno.

Uma justificativa para os valores apresentados acima é que, no verão, a alta temperatura, juntamente com a redução da umidade relativa do ar e da precipitação, não favoreça o aumento do número de casos notificados de pneumonia no município de Campina Grande. Percebe-se um aumento de casos da doença no início do período chuvoso, ou seja, à medida que a temperatura está baixando e, a umidade relativa do ar e a precipitação aumentando, o número de casos registrados dessa doença começa a aumentar.

A alta temperatura, a baixa umidade relativa do ar e a escassez de chuvas, durante os meses de setembro a dezembro, período que corresponde à primavera, parecem contribuir discretamente na redução do número de casos no decorrer dessa estação; contudo, observa-se, um pequeno aumento na incidência da doença, no início desse período.

Analisando-se as taxas de internações por pneumonia para $\mathrm{o}$ município de Campina Grande, mensalmente, há um comportamento que se repete nos anos avaliados, em que as menores taxas de internações são percebidas nos meses de verão, após o qual se elevam durante o outono, tendo seu pico observado no decorrer dos meses de inverno, decaindo novamente durante a primavera. Novamente os achados desta pesquisa se alinham aos do estudo de Pontes et al. (2016).

Para as internações gerais por pneumonia, as taxas de internação de crianças menores de cinco anos com essa patologia tiveram uma distribuição semelhante durante os meses do ano, sendo que no verão há um índice mais baixo de internações, com um aumento significativo durante o outono e seu pico máximo sendo claramente observado nos meses de inverno. Tal fato evidencia que durante o período de baixas temperaturas e elevada umidade do ar há um aumento das taxas de internações, decaindo novamente na primavera, com a retomada lenta da elevação da temperatura do ar.

$\mathrm{O}$ estudo demonstra que a temperatura média e a umidade relativa do ar têm influenciado os casos acometidos de pneumonia na cidade de Campina Grande, durante o período analisado, reforçando a tese de que mudanças bruscas de temperatura comprometem $\mathrm{o}$ funcionamento dos cílios responsáveis pela filtragem do ar aspirado, o que acarreta em uma maior exposição aos micro-organismos causadores da doença.

Mold (2008) ressalta que a redução da umidade relativa do ar a valores abaixo de $30 \%$ é considerada de risco para a integridade das vias aéreas, dificultando a homeostase interna do aparelho respiratório. Nos meses de chuva, em contraposição à problemática vivenciada nos meses de seca, a alta umidade relativa do ar, o aglomerado de pessoas aliada ao maior tempo de permanência nos ambientes internos, ao menor arejamento e exposição ao sol dos espaços domiciliares, com consequente crescimento de mofo e fungos, são fatores que podem contribuir para o aumento das DR, especialmente as alérgicas. 
Os resultados do presente trabalho são semelhantes e corroboram com o estudo de Mendonça (2000) que demonstrou a relação da temperatura com as doenças respiratórias, observando em seu estudo que quando as temperaturas estão baixas ocorre aumento na prevalência de doenças como bronquite, bronquiolite, bronquite crônica, asma e pneumonia.

Murara et al. (2013), também encontraram resultados semelhantes em seu estudo, retratando significância estatística para a variável temperatura do ar, o que demonstra que essa variável meteorológica influencia no comportamento das doenças respiratórias em geral e especialmente pneumonia.

O coeficiente de correlação de Pearson a um $\mathrm{p}<0,05$ entre os casos acometidos de pneumonia e as variáveis meteorológicas precipitação, temperatura do ar e umidade relativa do ar estão expostos na Tabela 5.

Tabela 5 - Coeficiente de Correlação de Pearson (r) e Coeficiente de Determinação em Campina Grande, PB - valores globais mensais

\begin{tabular}{c|c|c|c}
\hline Variáveis Meteorológicas & Pneumonia & & \\
\hline & $\mathrm{r}$ & $\mathrm{R}^{2}$ & Classificação \\
\hline Precipitação & 0,31 & 0,09 & Média \\
\hline Temperatura & $-0,71$ & 0,51 & Forte \\
\hline Umidade Relativa & 0,47 & 0,22 & Média \\
\hline
\end{tabular}

Fonte: Dados da pesquisa, 2017.

Como se nota, os casos de pneumonia na cidade de Campina Grande são sofrem influência da temperatura do ar. As evidências encontradas neste estudo reforçam o argumento defendido por Sette, Ribeiro e Silva (2012) quando analisaram a relação de doenças respiratórias na área urbana de Londrina/PR, Brasil e demonstraram a relação existente entre a temperatura e as internações hospitalares. Reforça, no caso específico da cidade de Campina Grande, PB, que os casos de pneumonia aumentam na medida em que a temperatura do ar diminui.

Em relação à variável umidade relativa do ar é possível observar que apresenta correlação positiva média $(\mathrm{r}=0,47$ e $\left.\mathrm{R}^{2}=0,22\right)$ para os casos de pneumonia. Esses resultados indicam que, à medida que a umidade relativa do ar aumenta, ocorre um aumento no número de pneumonia.

$\mathrm{Na}$ pesquisa realizada por Pontes et al. (2016) verificou-se que no verão há um índice mais baixo de internações por pneumonia com um aumento significativo durante o outono e seu pico máximo sendo claramente observado nos meses de inverno. Tal fato evidencia que durante o período de baixas temperaturas e elevada umidade do ar há um aumento das taxas de internações decaindo novamente na primavera, com a retomada lenta da elevação da temperatura do ar.

Em relação à variável precipitação a mesma apresentou correlação positiva média para pneumonia $\left(\mathrm{r}=0,31\right.$ e $\left.\mathrm{R}^{2}=0,09\right)$. $\mathrm{O}$ estudo demonstra que a temperatura média e a umidade relativa do ar têm influenciado os casos acometidos de pneumonia na cidade de Campina Grande, PB, durante o período analisado, reforçando a tese de que mudanças bruscas de temperatura comprometem $\mathrm{o}$ funcionamento dos cílios responsáveis pela filtragem do ar aspirado, o que acarreta em uma maior exposição aos microorganismos causadores da doença.

\section{CONSIDERAÇÕES FINAIS}

As principais contribuições do estudo apontam para uma realidade que vem a cada dia se mostrando relevante dentro do contexto da gestão de saúde pública, especialmente no que se refere à interferência das variações climatológicas e 
os supostos efeitos na saúde da população. No caso específico do gap que se procurou investigar pode-se inferir que:

- As doenças respiratórias são muito comuns e as formas de contraí-las também são muito abrangentes, por isso torna-se imprescindível realizar estudos regionalizados, além de identificar possíveis fatores, como as alterações climáticas como forma de ressaltar a importância de avaliar o efeito do clima na saúde humana.

- A faixa etária predominante para pneumonia foi aquela inferior a 5 anos de idade, seguidos da faixa etária de 60 anos ou mais, ocorrendo no município estudado.

- O estudo demonstra que a temperatura do ar e a umidade relativa do ar têm influenciado os casos acometidos de pneumonia com maior frequência na cidade de Campina Grande, PB, durante o período analisado.

As implicações encontradas apontam que quanto maior a umidade relativa do ar, e menor a temperatura do ar, mais casos de internações hospitalares por pneumonia ocorrem. Denotam a importância de conhecer a sazonalidade de certas doenças no intuito de permitir que os gestores de saúde tomem medidas de prevenção e promoção da saúde da população, principalmente quando se trata de faixas etárias com características conhecidas e que possam receber tratamento específico, reduzindo assim a necessidade de intervenções hospitalares conforme defendem Sette, Ribeiro e Silva (2012), Pontes et al. (2016), Mandú et al. (2019).

A limitação do estudo concentra-se no fato relacionado às subnotificações das doenças, falta de oportunidade no registro, atualização dos dados, diagnósticos incorretos, dentre outros, que podem ocorrer junto às repartições responsáveis pelo fornecimento de informações de saúde. Essa realidade em qualquer fase do sistema leva a um problema na disseminação da informação analisada, dificultando assim que a sociedade e os profissionais da saúde tenham as informações imprescindíveis para uma ação adequada e eficiente.

Outras doenças respiratórias e elementos meteorológicos poderiam ter sido utilizados no intuito de se ter um panorama mais amplo da realidade acerca da correlação entre essas variáveis e seu impacto na saúde pública.

\section{AGRADECIMENTO}

Ao Conselho Nacional de Desenvolvimento Científico e Tecnológico pelo apoio concedido durante a realização da pesquisa na etapa do doutoramento da primeira autora.

\section{REFERÊNCIAS}

ALVES, T. L. B.; AZEVEDO, J. V. V. de; SANTOS, C. A. C. dos; AZEVEDO, P. V. de. Influência das variações climáticas na ocorrência de doenças das vias aéreas superiores no município de Monteiro - PB. Ciência e Natura, Santa Maria, v. 37 n. 4 set-dez. p. 433-450, 2015.

AMORIM, J. R. G; OLIVEIRA, A. M.; NEVES. D; OLIVEIRA, G. P. Associação entre variáveis ambientais e doenças respiratórias (asma e bronquite) em crianças na cidade Macapá-AP no período de 2008 a 2012. Planeta Amazônia: Revista Internacional de Direito Ambiental e Políticas Públicas, Macapá, n. 5, p. 141153, 2013.

AYOADE, J. O. Introdução à Climatologia para os trópicos. In: J. O. Ayoade, Tradução de Maria Juraci Zani dos Santos. 13. ed. Rio de Janeiro: Bertrand Brasil, 2010. 
AZEVEDO, J. V. V.; SANTOS, C. A.C.; ALVES, T. L. B.; AZEVEDO, P. V.; OLINDA, R. A. Influência do clima na incidência de infecção respiratória aguda em crianças nos municípios de Campina Grande e Monteiro, Paraíba, Brasil. Rev. bras. meteorol. vol.30, n. 4, out./ dec. 2015.

AZEVEDO, J. V. V.; SANTOS, C. A.C.; SILVA, M. T.; OLINDA, R. A.; SANTO, D. A. S. Análise das variações climáticas na ocorrência de doenças respiratórias por Infuenza em idosos na região metropolitana de João Pessoa - PB. Revista Sociedade \& Natureza., Uberlândia, v.29, n. 1, p. 123135, mai/ago, 2017.

BRASIL. Ministério da Saúde. Secretaria de Atenção à Saúde. Departamento de Atenção Básica. Doenças respiratórias crônicas / Ministério da Saúde, Secretaria de Atenção à Saúde, Departamento de Atenção Básica. - Brasília: Ministério da Saúde, 2010.

CALLEGARI-JACQUES, S. M. Bioestatística - Princípios e Aplicações. Editora Artmed, 2009.

CASTRO, H. A. de; CUNHA, M. F. da; MENDONÇA, G. A. e S.; JUNGER, W. L. J.; CUNHA-CRUZ, J.; LEON, A. P de. Efeitos da poluição do ar na função respiratória de escolares, Rio de Janeiro, RJ.

Revista de Saúde Pública, v.43, n.1, pp. 26-34, 2009.

CHAN, P.W.; GOH, A.Y.; CHUA, K.B; KHARULLAH, N.S.; HOOI, P.S. Viral aetiology of lower respiratory tract infection in young Malaysian children. Paediatr Child Health 1999; 35: 287-290.

CHEN, N.; CHEN, M. GUO, C.; CHEN, K.; SU, H. Precipitation Increases the Occurrence of Sporadic Legionnaires' Disease in Taiwan. PLos One, v. 9, n. 12, 2014.
CONCEIÇÃO, R. S.; SILVEIRA, G. S. P.; VEIGA, A. J. P.; MATTA, M. B. M. A temperatura do ar e sua relação com algumas doenças respiratórias em vitória da conquista - BA. Revista Eletrônica Geoaraguaia. Barra do Garças, MT, v. 5, n. 2, p. 69-81, Jul./Dez., 2015.

COSTA, E. de O.; SILVA, C. S.; SOARES, M. E. S. M.; SILVA, R. G. da S.; AMARAL, P. B. Análise do Tempo de Internação de Crianças com Pneumonia em Hospital Público de João Pessoa-PB. Revista Brasileira de Ciências da Saúde, v. 18, n. 2, p.147-150, 2014.

FRANCO, R. G. S. Estudo da Relação dos casos de asma e bronquite em crianças menores de 5 anos $e$ variáveis meteorológicas no município de Uberlândia-MG. Dissertação apresentada ao Programa de Pós-Graduação Mestrado Profissional em Saúde Ambiental e Saúde do Trabalhador do Instituto de Geografia da Universidade Federal de Uberlândia, 2017.

GHALHARI, G.F.; MAYVANEH, F. Effect of air temperature and Universal Thermal Climate Index on respiratory diseases mortality in Mashhad, Iran. Archives of Iranian Medicine, v. 19, n. 9, p. 618-624, 2016.

GOUVEIA, N.; MENDONÇA, G. A. S.; LEON, A. P.; CORREIA, J. E. M.; JUNGER, W. L.; FREITAS, C. U.; DAUMAS, R. P.; MARTINS, L. C.; GIUSSEPE, L.; CONCEIÇÃO, G. M. S.; MANERICH, A.; CUNHA-CRUZ, J. Poluição do ar e efeitos na saúde nas populações de duas grandes metrópoles brasileiras. Epidemiologia e Serviços de Saúde, v. 12, n. 1, 29-40, 2003.

IBGE. Instituto Brasileiro de Geografia e Estatística. Localização do Município de Campina Grande, PB. Disponível em: https://cidades.ibge.gov.br/painel/painel.ph $\mathrm{p}$ ? codmun $=2504009$. Acesso em: 17 nov. 2017. 
MANDÚ, T. B.; GOMES, A. C. S.; SANTOS, M. S.; ALFAIA, V. M. Efeito de condições meteorológicas em doenças respiratórias em capitais de diferentes dimensões no Norte e Nordeste do Brasil. REGNE, vol. 5, $\mathrm{n}^{\circ}$ 1, p. 86-100, 2019.

MARTINS, A. L O; TREVISOL, F. S. Internações hospitalares por pneumonia em crianças menores de cinco anos de idade em um hospital no Sul do Brasil. Revista AMRIGS, Porto Alegre, v. 57, n. 4, p. 304308, out/dez. 2013.

MIRANDA, M. J. Análise temporal das internações por gripe e pneumonia associadas às variáveis meteorológicas no município de São Paulo, SP. Revista do Instituto Geológico, São Paulo, v. 37, n. 2, 61-71, 2016.

MOLD. Una breve guía para el moho la humedad y su hogar. Agency Washington, DC, United States Environmental Protection. 2008. Disponível em: http://www.epa.gov/mold/moldresources.ht ml. Acesso em:18/04/2016.

MOTA, S. Saúde Ambiental. In: ROUQUAYROL, M. Z; SILVA, M. G. C. Epidemiologia e Saúde Pública. 7ed. Rio de Janeiro: Med book, 2013.

MOURA, M.; JUNGER, W. L.; MENDONCA, G. A. e S.; PONCE DE LEON, A. Qualidade do ar e transtornos respiratórios agudos em crianças. Revista de Saúde Pública, vol.42, n.3, p. 503-511, 2008 .

MURARA, P. G; MENDONÇA, M; BONETTI, C. O clima e as doenças circulatórias e respiratórias em Florianópolis/SC. Hygeia: Revista Brasileira de Geografia Médica e da Saúde, Uberlândia, v. 16, n. 9, p. 86-102, jun. 2013.

NASCIMENTO, L. F. C.; PEREIRA, L. A. A.; BRAGA, A. L. F.; MÓDOLO, M. C. C.;
CARVALHO, JÚNIOR, J. A. Efeitos da poluição atmosférica na saúde infantil em São José dos Campos, SP. Revista de Saúde Pública, v. 40, n.1, p. 77-82, 2006.

NATALI, R. M. T.; SANTOS, D. S. P. S. dos; FONSECA, A. M. C. da; FILOMENO, G. C. de M. FIGUEREDO, A. H. A.; TERRIVEL, P. M.; MASSONI, K. M.; BRAGA, A. L. F. Perfil de internações hospitalares por doenças respiratórias em crianças e adolescentes da cidade de São Paulo, 2000-2004. Revista Paulista de Pediatria, v.29, n. 4, p. 584-590, 2011.

OLULEYE, A.; AKINBOBOLA, A. Malaria and pneumonia occurrence in Lagos, Nigeria: Role of temperature and rainfall. African Journal of Environmental Science and Technology, vol. 4(8), p. 506-516, August 2010.

OMS. Organização Mundial de Saúde. 2012. Disponível em: www.paho.org/bra/ Acesso em: 15 ago. 2015.

PONTES, C. C.; LEITE, M. L.; GAVÃO, N.; VIRGENS, F. J. S. Efeitos do clima na saúde: análise das internações de crianças menores de cinco anos por pneumonia no município de ponta grossa - PR. Revista Brasileira de Climatologia, v.18, n.12, 2016.

QIU, H.; SUN, S.; TANG, R.; KING-PAN, C.; TIAN, L. Pneumonia Hospitalization Risk in the Elderly Attributable to Cold and Hot Temperatures in Hong Kong, China. American Journal of Epidemiology, v.184, Issue 8, 15 October 2016, p. 570-578.

RUDAN, I.; O'BRIEN, K.L.; NAIR, H.; LIU, L.; THEODORATOU, E.; QAZI, S.; LUKŠIĆ, V.; WALKER, C.L.F.; BLACK, R.B.; CAMPBELL, H. Epidemiology and etiology of childhood pneumonia in 2010: estimates of incidence, severe morbidity, mortality, underlying risk factors and causative pathogens for 192 countries. 
Journal of Global Health, v.3, n. 1, p. 10401, 2013.

ROUQUAYROL， M. Z; ALMEIDA FILHO, N. Epidemiologia e Saúde Pública. 6ed. Rio de Janeiro: Medsi, 2013.

SALVI, S. Health effects of ambient air pollution in children. Paediatric Respiratory Reviews, 8, p. 275-280, 2007.

SANTOS, D. A.S.; AZEVEDO, P. V.; OLINDA, R.; SOUSA, A.; AZEVEDO, J. V. V. SILVA, M. S.; SILVA, F. P. Influência das variáveis climáticas na hospitalização por pneumonia em crianças menores de cinco anos em RondonópolisMT. Revista Brasileira de Geografia Física, v.09, n. 02, p. 413-429, 2016.

SETTE, D. M; RIBEIRO, H.; SILVA, E. N. $\mathrm{O}$ índice de temperatura fisiológica equivalente (PET) aplicado a Londrina, PR., e sua relação com as doenças respiratórias. Revista Geonorte, Edição Especial 2. p. 813-825, 2012.

SILVA, S. R. da; MELLO, L. M. de; SILVA, A. S. da; NUNES, A. A. Impacto da vacina antipneumocócica 10 -valente na redução de hospitalização por pneumonia adquirida na comunidade em crianças. Revista Paulista de Pediatria, v. 34, n. 4, p. 418-424, Dez. 2016.

SOUSA, C. A. de; CÉSAR, C. L. G.; BARROS, M. B. de A.; CARANDINA, L.; GOLDBAUM, M.; PEREIRA, J. C. R. Doenças respiratórias e fatores associados: estudo de base populacional em São Paulo, 2008-2009. Revista de Saúde Pública, v. 46, n. 1, p. 16-25, 2012.
SOUZA, A. de; ARISTONE, F.; SANTOS, D. A. S.; FERREIRA, W. M. Impacto de Variações Climáticas em Casos de Asma em Campo Grande, MS. Engenharia Ambiental - Espírito Santo do Pinhal, v. 11, n. 2, p. 142-153, jul./dez. 2014.

STRACHAN, D.P; SANDERS, C. H. Damp housing and childhood asthma; respitatory effects of indoor air temperature and relative humidity. J. Epidemiol. Community Health, v. 43, n.7, 1989.

TRAVASSOS, K. D. Crescimento e desenvolvimento de variedades de girassol irrigado com água salina. Irriga, Botucatu, Edição Especial, p. 324-339, 2012.

XU, Y.; ZHU, L.; XU, D.; TAO, X.; LI, S.; TANG, L.; CHEN, Z. Epidemiological characteristics and meteorological factors of childhood Mycoplasma pneumoniae pneumonia in Hangzhou. World J. Pediatr, v.7, $\mathrm{n}^{\mathrm{o}}$ 3, August 15, 2011.

XU, Z.; LIU, Y.; MA, Z.; LI, S. HU, W.; TONG, S. Impact of temperature on childhood pneumonia estimated from satellite remote sensing. Environmental

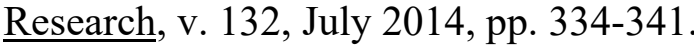

$\begin{array}{lr}\text { WHO }- & \text { WORLD HEALTH } \\ \text { ORGANIZATION. Pneumonia. } & 2014 . \\ \text { Disponível } & \end{array}$
http://www.who.int/mediacentre/factsheets /fs331/en/. Acesso em: 19 ago. 2015.

WEBER, M.W.; DACKOUR, R.; USEN, S. et al. The clinical spectrum of respiratory syncytial virus disease in The Gambia. Pediatr Infect Dis J,17, p. 224-230, 1998.

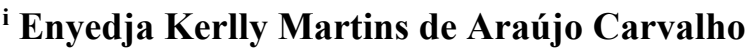

Professora da Faculdade São Francisco da Paraíba. Enfermeira pela Universidade Estadual da Paraíba (UEPB), Mestra e Doutora pelo PPGRN UFCG.

ii Renilson Targino Dantas

Professor-doutor da Universidade Federal de Campina Grande.
} 


\section{iii José Ribamar Marques de Carvalho}

Mestre em Ciências Contábeis. Doutor pelo Programa de Pós-Graduação em Recursos Naturais PPGRN/UFCG. Professor do Curso de Ciências Contábeis da UFCG. Pós-doutorando em Ciências Contábeis pelo PPGCON - Escola de Negócios da UNISINOS.

${ }^{\text {iv }}$ Este artigo é parte integrante da tese de doutorado da primeira autora. A estrutura completa do modelo com as respectivas tabelas das variáveis do estudo encontra-se com a autora principal. Tais informações poderão ser disponibilizadas. Optou-se pela não inclusão em razão da limitação de espaço. 